\title{
EL PROYECTO GRIALE PARA LA IRONÍA EN ESPAÑOL: CONCEPTOS PREVIOS
}

\author{
Leonor Ruiz Gurillo, Carmen Marimón, \\ Xose A. Padilla y Larissa Timofeeva \\ Universidad de Alicante. Grupo GRIALE \\ Leonor.Ruiz@ua.es,marimon@ua.es,Xose.Padilla@ua.es, Timofeeva@ua.es
}

\begin{abstract}
Resumen
The aim of this article is to present an outline of the main working lines of GRIALE researching group. This group, integrated by members of the Department of Hispanic Philology from the Universidad de Alicante, has as first task the study of verbal irony in Spanish as well as its application to the teaching of Spanish as LE/L2. Starting form a pragmatic model as the echoic interpretation theory (Sperber and Wilson, 1978), we analyze irony as a variety of echoic mention, we also present a first proposal of different kinds of verbal irony in relation with text typology and we also point out some verbal procedures used to indicate the presence of irony in an utterance or in a written text.
\end{abstract}

\section{Introducción}

El grupo GRIALE inició sus investigaciones a finales de 2002. Dirigido por Leonor Ruiz Gurillo, está compuesto por diversos profesores del Área de Lengua Española de la Universidad de Alicante: Belén Alvarado, Elisa Barrajón, Jorge Fernández, Carmen Marimón, José Joaquín Martínez, Xose A. Padilla, Herminia Provencio, Francisco Reus, Susana Rodríguez, Santiago Roca, Isabel Sánchez, Isabel Santamaría y Larissa Timofeeva. La formación multidisciplinar de sus miembros resulta clave para afrontar los diversos trabajos de investigación que se han propuesto. Actualmente contamos con un Proyecto de Investigación subvencionado por el Ministerio de Educación y Ciencia (HUM2004-01318/FILO) para los años 2004-2007, que se encarga de la descripción, del análisis y de las aplicaciones de la ironía en español actual desde el punto de vista pragmático. El fin último es elaborar una tipología de enunciados irónicos que pueda aprovecharse en la enseñanza del español como lengua extranjera (E/LE). En este sentido, nuestra aplicación, que se ajusta a las exigencias del Programa MARCO de la Unión Europea, tiene en cuenta los niveles competenciales (C2 usuario competente, $\mathrm{C} 1$ usuario competente, $\mathrm{B} 2$ usuario independiente) en los que es posible enseñar la ironía verbal y, de acuerdo con ello, se elaborarán ejercicios y actividades. El trabajo sobre uno de los significados más pragmáticos, el de la ironía verbal, se apoya sobre corpora reales orales y escritos que permiten comprobar la validez de las hipótesis teóricas propuestas. Tras el análisis y la descripción de su funcionamiento, se establecerá un sistema de reconocimiento que será aplicable al conjunto del corpus. Ello permitirá establecer una 
tipología de enunciados irónicos y, en la última fase del Proyecto, aplicar tal tipología a la enseñanza del E/LE.

Tal vez, la ironía constituye, junto con la metáfora y la representación del discurso, el fenómeno pragmático por excelencia. El Proyecto de Investigación que presentamos se centra en los aspectos más pragmáticos del significado al haber elegido la ironía como centro de las indagaciones, pues ésta constituye "la piedra de toque de toda teoría que pretenda explicar la relación entre significado y uso" (Reyes et alii, 2000: 144). Frente a otras propuestas llevadas a cabo en el ámbito hispánico, nuestro Proyecto no se queda en la mera información teórica y en la descripción de enunciados elegidos ad hoc, sino que convierte el manejo de corpora reales y la aplicación didáctica de los resultados en sus pilares básicos.

Así, para el análisis de la ironía se están empleando tanto muestras orales como escritas. Las muestras orales proceden, fundamentalmente, de los corpora ALCORE, COVJA y Val. Es.Co, a cuyos grupos de trabajo pertenecemos muchos de los integrantes de GRIALE. Al trabajo del Área de Lengua Española de la Universidad de Alicante están vinculados los corpora reales del español COVJA y ALCORE. El Proyecto COVJA (Corpus Oral de la Variedad Juvenil Universitaria del Español Hablado en Alicante), dirigido por Dolores Azorín Fernández y Juan Luis Jiménez Ruiz, cuenta con una publicación editada en 1999. En cuanto al proyecto ALCORE (Alicante-Corpus Oral del Español), dirigido por Dolores Azorín Fernández, editó sus resultados en CD-Rom en 2002. Asimismo, el corpus Val.Es.Co. (Valencia, Español Coloquial), dirigido por Antonio Briz de la Universitat de València, dispone hasta el momento de dos volúmenes, Briz (Coord.) (Grupo Val.Es.Co.) (1995) y Briz y Grupo Val.Es.Co. (2002). Por lo que se refieren a las muestras escritas, proceden fundamentalmente de la prensa y, en particular de las editoriales, los artículos de opinión y las columnas. También se consultan otros corpora disponibles a través de la red como el CREA (http://www.rae.es) o el Corpus del Español (http://www. corpusdelespanol.org).

Los resultados que se obtengan se aplicarán en diversas actividades de E/LE que se difundirán tanto en formato libro como a través de nuestra página web (http://www.griale. ua.es), o por medio de otras herramientas virtuales como la Didactiteca del Instituto Cervantes (http://www.cvc.cervantes/aula/didactired).

Desde hace unos meses GRIALE se encuentra inmerso en la primera fase del proyecto, de manera que está analizando los diversos modelos teóricos construidos para la ironía verbal. Hasta el momento, hemos observado que, aunque se puede explicar como un eco de otro enunciado que se menciona (mención ecoica), como propusieron Sperber y Wilson en 1978 y como han manifestado otros autores como Ducrot [Ducrot et alii (1980), Ducrot (1986), (1988), (1996) y (2003)], Clark y Gerrig (1984), Reyes (1994) y (2002) o Torres Sánchez (1999), existen diversos tipos de eco que repercuten en el contexto lingüístico, el contexto situacional o el contexto sociocultural (como examinaremos en el punto 2). De igual modo, puede diferenciarse de antemano una ironía focalizada, que parece más relacionada con textos orales y en los que resulta posible extraer el eco del que se hace ironía, y una ironía continuada, más propia de los textos escritos y donde se propone un entorno irónico (punto 3). Por otra parte, la ironía hace uso de diversos indicadores que la favorecen, como los gestos o la mímica, las palabras marcadas ideológicamente o la fraseología (punto 4). En este sentido, uno de los retos actuales consiste en sistematizar los recursos que facilitan la interpretación irónica por parte del oyente. Para ilustrar tales hechos, emplearemos a con- 
tinuación ejemplos diversos que proceden de nuestro corpus y que, por tanto, provienen de textos coloquiales, literarios o periodísticos.

\section{La mención ecoica: una explicación para la ironía}

Desde que la pragmática decidió tratar el tema de la ironía, uno de los objetivos principales de los investigadores ha sido desmarcarse de las definiciones tradicionales. Para la retórica clásica, la ironía era un tropo o figura de pensamiento ${ }^{1}$ destinado a expresar lo contrario de lo que se afirma. Así, en un enunciado como:

(1)

Rechazado por él, te pusiste a vivir con tu compañero, hombre nobilísimo, Metello (Cat., $1,8,19)^{2}$

el interpretante del mensaje -afirmaban los retóricos- entiende que Metello no es noble, y no entiende que sí lo es, porque el hablante hace una burla y cambia el significado convencional de las palabras.

La pragmática ${ }^{3}$ propone aparcar las definiciones de la retórica y utilizar el concepto mención ecoica. Así define Reyes (1994: 50) el concepto:

[...] el hablante se hace eco del contenido de otro enunciado deformándolo, exagerándolo o modificándolo burlonamente, con la intención de mostrar una actitud negativa ante ese enunciado o ante su autor.

y añade:

La ironía es una figura, pero debemos corregir su definición tradicional: [como] «figura que consiste en dar a entender lo contrario de lo que se dice» (Reyes, 1994: 52).

Es decir, que en un ejemplo como:

Mujer: ¿Me quieres, cariño? dímelo otra vez, venga

Marido: Sí, mi amor, te adoro

los pragmatistas opinarían que el segundo hablante (el marido) no busca expresar que ya no quiere a su esposa, sino que está cansado de que ella se lo pregunte constantemente.

En las reuniones de nuestro grupo, la cuestión del enfrentamiento entre retóricos y pragmatistas ha sido el tema de numerosas sesiones. No hemos llegado todavía a conclusiones definitivas, pero, sin negar que la contradicción entre lo que se dice y lo que se intenta decir sea un factor importante, pensamos que el concepto mención ecoica podría ayudar a definir

1 De las cinco fases de elaboración del discurso retórico -inventio, dispositio, elocutio, memoria y actio-, la ironía forma parte de la parte elocutiva, aquella dedicada a poner en funcionamiento los recursos lingüístico-verbales adecuados para expresar los contenidos del discurso y que éste realice la función que el hablante desea.

2 Hemos obtenido este ejemplo de Quintiliano (IX, 2, 45).

3 Véase, por ejemplo, Sperber y Wilson (1978) o Reyes (1994). 
de manera más adecuada qué entendemos por ironía, aunque sin despreciar las sugerencias que nos brinda la retórica.

El concepto mención ecoica, sin embargo, presenta también algunos problemas, sobre todo, cuando el eco no es completamente transparente. En algunos ejemplos, es fácil aceptar que la ironía es un eco porque las palabras retomadas repiten parte del discurso anterior; en otros, no lo es tanto, porque lo ecoizado pertenece a los conocimientos compartidos por los hablantes sobre el mundo o sobre ellos mismos.

Muchos de los enunciados que hemos analizado en las reuniones (principalmente los coloquiales) han representado un difícil problema tanto en la interpretación irónica como en su supuesta ecoicidad. Sirvan como botón de muestra los siguientes:

(3)

B: ¿cuántos años tiene la tía?

A: treinta y nueve ${ }^{4}$

$\mathrm{D}$ : igual que nosotros/ to- tiene la misma edad

([H.38.A.1], líneas, 175-177, Briz y grupo Val.Es.Co, 2002: 54)

El ejemplo (3), a pesar de estar marcado como irónico por el transcriptor (véase nota 4), es difícil de interpretar como tal si no tenemos acceso a la información pragmática compartida. En este caso, que los hablantes tienen alrededor de veinte años y que, aparentemente, las mujeres de treinta y nueve no les resultan atractivas.

Veamos ahora el ejemplo (4):

(4)

$\mathrm{C}$ : el que era capaz de- de montar una frase/ y hasta cantar una canción en- con eructos era [el $\operatorname{Mosca}^{5}$ ¿eh?]

D: $\quad$ [el Mosca]/ el Mosca sí

A: ese era un cerdo

D: [(RISAS)]

B: [(RISAS)]

C: [(RISAS)] escupir y eructar $\uparrow$ era algo $\rightarrow$ era algo innato en él

D: $[$ y y y $=$ ]

B: [caballeros así ya no salen]

D: = y Emiliano se mos- amos- a veces se mosqueaba con él

([H.38.A.1], líneas, 530-538, Briz y grupo Val.Es.Co, 2002: 64)

En esta ocasión, parece bastante claro que estamos ante un enunciado irónico (el Mosca lógicamente no es un caballero). El problema, sin embargo, radica en la dificultad para reconocerlo como un eco (en el texto no hay elementos a los que remitir directamente).

Todas estas razones nos llevan a pensar que es dificil calificar un enunciado como irónico si no se definen con mayor nitidez los límites de la ecoicidad. 
Para esclarecer cuál es el origen de un determinado eco irónico, hemos decidido definir tres tipos de contexto de remisión que derivan en cierto modo de los tres componentes que Dik (1989) definió como parte del concepto información pragmática ${ }^{6}$, que son:

a) contexto lingüistico ${ }^{7}$ : el cotexto, la información verbal identificable en el discurso previo,

b) contexto situacional: la situación externa, el escenario que rodea a los hablantes,

c) contexto sociocultural: vivencias y conocimiento del mundo, las características naturales, sociales, culturales, etc. que conforman nuestra experiencia vital.

Atendiendo a tales diferencias, los enunciados irónicos pueden ser definidos como ecos, pero la mención será más o menos transparente según nos situemos en uno u otro tipo de contexto.

Muchos enunciados irónicos coloquiales, como los que hemos visto, son opacos a la interpretación del investigador, porque éste no es capaz de restituir la información pragmática que en aquel momento compartían los hablantes, ya que el eco se sitúa en el complejo contexto sociocultural, una suma indefinida de todos los posibles esquemas mentales ${ }^{8}$ (particulares y generales) de los hablantes.

Ahora bien, tal aproximación no es suficiente para discriminar la ironía y habremos de tomar en consideración otros factores, como las circunstancias que rodean a la comunicación.

\section{La ironía y el modo de comunicación: ironía focalizada e ironía continuada}

Estas primeras aproximaciones a los enunciados irónicos y a sus posibilidades de análisis y clasificación nos han llevado a observar que, en general, el concepto de mención ecoica y, en consecuencia, la interpretación de la ironía, no se puede aplicar en los mismos términos cuando nos referimos a enunciados orales o cuando analizamos textos escritos. En estos primeros tanteos hemos podido observar que, mientras en los intercambios orales el eco tiende a ser de lo dicho previamente por el interlocutor y en ese caso es relativamente sencillo rescatar el enunciado ecoizado, en los textos escritos la ironía se produce, en numerosas ocasiones, en relación con algo que no forma parte del intercambio comunicativo inmediato -pues éste no existe-- sino que se supone un eco de algo a lo que el hablante remite y que el destinatario debe rescatar en relación ahora con el conocimiento compartido de ambos. Veamos qué ocurre en los ejemplos (5) y (6), el primero oral y el segundo escrito:

6 Los tres componentes son: (a) general: comprende el conocimiento del mundo, de sus características naturales, etc.; (b) situacional: abarca el conocimiento derivado de los que los interlocutores perciben durante la interacción; y (c) contextual: incluye lo que deriva de las expresiones lingüísticas intercambiadas en el discurso inmediatamente precedente (véase Escandell, 1996: 31).

7 Estos conceptos han sido desarrollados en Padilla (2001 y 2005).

8 Es decir, vivencias, costumbres, situaciones que atañen a un número más o menos amplio de hablantes. Yule (1996: 85) habla de los esquemas (véase también Van Dijk, 1977) como estructuras de conocimiento preexistentes en la memoria que reflejan "the normal expected patterns of things", y establece dos tipos: (a) los marcos (o frames) (véase también Bateson, 1972 y Goffman, 1981) que son estructuras de conocimiento preexistentes para describir patrones estáticos o fijos; y (b) los guiones (scripts) (véase también Abelson y Schank, 1977) que son estructuras de conocimiento preexistente para interpretar secuencias dinámicas o acciones. 
(5)

B: ¿Emiliano $\uparrow$ alto $\uparrow$ de qué $\downarrow$ ?

A: ¿có[mo sería de alto?]

B: [ni física ni mental]mente es alto $\downarrow$ nano

D:

$\S$ como yo/ como nosotros/ más o menos

A: entonces muy alto $\uparrow$ el Conejo/// pero el Conejo nunca ha ido por el Carmen $9 /$ que yo sepa§

D: $\quad \S[\mathrm{YA}]$

([H.38.A.1], líneas, 150-157, Briz y grupo Val.Es.Co, 2002:54)

(6)

Panotxo Murziazoa (La Verdad Digital, 21-I-2004)

En el ejemplo (5), procedente del corpus coloquial de Val.Es.Co., el hablante A retoma un elemento del enunciado, alto, al que han hecho referencia todos los hablantes en el transcurso de la conversación, y emite su enunciado, entonces muy alto, con el que responde irónicamente al intercambio mantenido en relación con la altura del individuo objeto de la conversación. Por tanto, hay mención de algo dicho previamente.

Sin embargo, en el ejemplo (6), que es el título de un artículo de opinión aparecido en el diario $L a$ verdad digital, no hay ningún enunciado al que remitirse para recuperar el sentido irónico de la frase. Para que esto suceda, el emisor confía en que el conocimiento enciclopédico de los posibles destinatarios así como su competencia lingüística y sociolingüística sean suficientes para interpretar Panotxo murziazoa como un enunciado irónico. Así, espera que los lectores pongan en relación las alteraciones ortográficas y morfológicas sufridas por las palabras panocho y Murcia con la ortografía y la morfología más reconocible de otra lengua del estado, el euskera; además, como el periódico se edita en Murcia, el emisor supone también que el destinatario está medianamente familiarizado con cierta polémica lingüística en torno al panocho -recreación del habla murciana-. Y que, como ciudadano español, conoce también las dimensiones del conflicto político y lingüístico de Euskadi. Toda esa información, como mínimo, es la que se activa para entender Panotxo Murziazoa como una ironía sobre la «euskarización» del panocho y, desde luego, predispone al lector a leer irónicamente el artículo que encabeza. En este caso, por tanto, es el contexto sociocultural el que permite comprender la ironía.

Aunque, en efecto, no es posible adjudicar en exclusiva cada una de estas dos posibilidades de construir ironías al uso de un determinado canal, la tendencia nos está resultando innegable y es eso lo que nos ha llevado a tomar algunas decisiones. Por una parte, a reafirmarnos en la necesidad de utilizar material de análisis perteneciente a distintos modos comunicativos, tal y como detallábamos en la introducción, y, por otra, a intentar establecer una primera distinción teórica con la que trabajar estas formas diferentes de manifestación de la ironía. Por ello, diferenciamos dos tipos de ironía, focalizada y continuada.

La ironía focalizada es aquella que se produce en un enunciado concreto. Suele ayudarse de indicadores lingüísticos específicos y está vinculada habitualmente al contexto situacional y lingüístico, que es donde se localiza el enunciado que se menciona ahora como eco de aquél. 
La ironia continuada, por su parte, es aquella que no aparece necesariamente en un enunciado concreto, sino a lo largo de todo un texto. No siempre presenta indicadores lingüísticos específicos, pero viene favorecida por un entorno irónico que, según Utsumi (2000: 1783-1784), consiste en la expectativa del hablante, en la incongruencia entre la expectativa y la realidad y en una actitud negativa del hablante hacia su congruencia.

La relación de la ironía focalizada con el contexto lingüístico y situacional y de la ironía continuada con el contexto situacional y sociocultural es, en el momento en que nos encontramos de la investigación, una tendencia útil para discriminar los factores que intervienen en la ironía. Estamos seguros, sin embargo, de que en el desarrollo de nuestro trabajo nos será posible perfilar, completar y matizar nuestras propuestas iniciales.

En cualquier caso, a favor de este primer esbozo tipológico tenemos algunos conceptos básicos de la retórica clásica, en la que se diferenciaban también dos formas de ironía, la ironía como tropo y la ironía como figura. Cuando, en el proceso de construcción discursiva, el hablante se centraba ya en los aspectos lingüístico-verbales, debía elegir las palabras y recursos que hicieran el discurso adecuado, correcto, comprensible y bello. En este último caso, -el de la belleza-, el objetivo era engendrar admiración, atención y sorpresa en el oyente de manera que fueran las propias palabras las que, ahora, sirvieran a los fines discursivos. E1 ornatus, que es donde se encuentran esos recursos embellecedores, está organizado en dos grandes grupos: (A) In verbis singulis -para palabras aisladas-cuya máxima expresión son los tropos y (B) In verbis coniuctis -para palabras unidas- cuya principal expresión son las figuras. Pues bien, la ironía es considerada tanto un tropo, es decir, un recurso que se puede llevar a cabo únicamente con algún tipo de modificación en una palabra, como una figura, una modificación más compleja que afecta a un segmento mayor del discurso.

En el primer caso, la ironía se concretaba en una palabra aislada que suponía un desplazamiento semántico - en este caso en el sentido de expresar lo contrario de lo que se decía-. Estaríamos próximos a lo que nosotros hemos denominado una ironía focalizada. La ironía como figura, más difícil de concretar lingüísticamente, tendría que ver con el tono y la intención general del discurso tal. Así la explica Quintiliano:

En la figura de la ironía se trata del fingimiento de toda la intención, que se trasluce más que se manifiesta, de suerte que allí -en el tropo- las palabras son contrarias unas a otras, mientras aquí -en la ironía como figura- se contrapone el sentido a la expresión completa y a su tono, y a veces la configuración entera de un caso, hasta una vida ente$\mathrm{ra}$, puede tener ironía. [...] de suerte que lo mismo que una metáfora continuada forma alegoría, así forma el tropo de la ironía con todo su contexto esta correspondiente figura (Quint., IX, 2, 46).

Sería este caso el que denominamos ironía continuada para cuyo desarrollo es necesario un ámbito discursivo, sea este escrito u oral, que permite mantener ese tono irónico.

\section{Indicadores de la ironía}

La ironía necesita del destinatario que ha de entenderla y reconocerla, por lo que la colaboración entre hablante y oyente se convierte en imprescindible. Existe una relación directamente proporcional entre la opacidad ecoica del enunciado y la necesidad que tiene el hablante de utilizar determinados indicadores de su intención comunicativa. El hablante 
no necesita asegurarse una comprensión perfecta del significado irónico de sus palabras, pero sí debe confeccionar una guía, es decir, debe proporcionar las pistas suficientes para que el buen entendedor acierte. Entre los objetivos de nuestro proyecto se encuentra el de dilucidar qué procedimientos lingüísticos y no lingüísticos utiliza para indicar su intención y qué elementos señalan al destinatario la posibilidad de la interpretación de un enunciado en clave irónica. En este sentido, Schoentjes (2003) lleva a cabo una interesante aproximación a los indicadores de la ironía, que empleamos seguidamente ${ }^{10}$.

En primer lugar, se habla de la importancia de gestos y mímica ${ }^{11}$, ya que se considera que la ironía nace en el discurso oral. Asimismo, se subraya la importancia del tono irónico. En las reuniones de nuestro grupo estamos dedicando bastante tiempo al estudio de este indicador, que en opinión de algunos miembros constituye en muchos casos un elemento imprescindible para que se origine la ironía en el nivel oral. A favor de tal argumento contamos con la afirmación de Schoentjes (2003: 138) para el que un hablante cualquiera reconoce sin problemas una entonación irónica, pues las variedades entonativas las aprendemos desde pequeños, aún antes que el significado de las palabras.

Sin embargo, si nos enfrentamos a un texto escrito, el tono irónico que reconoce el destinatario se concreta en otros indicadores. En primer lugar, en la puntuación. Tal y como ocurre con otros indicadores lingüísticos, no existe, al menos en español, ningún signo de puntuación específico para los enunciados irónicos. Los signos de exclamación y los puntos suspensivos se usan a veces para desempeñar el papel de los indicadores no verbales en el lenguaje oral, aunque quizá las comillas sea el medio más utilizado para señalar el carácter irónico de algún elemento, como podemos observar en (7) y (8):

\section{(7)}

...porque, tal y como está el mundo de memo y de policial, raro será el texto que no "ofenda" a alguien, o ante el que algún paranoico no se dé por aludido (J. Marías, El País Semanal, 5-XI-2004).

(8)

Ya sería hora de que [...] los "delicados" se endurecieran un poquito la piel tan fina que se han colocado (J. Marías, El País Semanal, 5-XI-2004).

Un papel semejante al de los signos de puntuación cumplen las denominadas palabras de alerta. Su función es la de "exagerar la fuerza de un enunciado o volverlo sospechoso" (Schoentjes 2003: 143). Se trata del uso de palabras fuera de su registro, de algunos adjetivos elogiosos, etc., como se ilustra en (9):

10 Para el tema de los indicadores de la ironía también pueden consultarse los trabajos de Torres Sánchez (1999), Reyes (1994), así como Knox (1961), Muecke (1970) o Culler (1975).

11 Para Poyatos (1994: cap. 4), la comunicación posee una estructura tripartita (la denomina la triple estructura básica de la comunicación), compuesta del lenguaje, paralenguaje y kinésica. Según esta clasificación los gestos y ta mímica forman parte de la kinésica, el tono corresponde al paralenguaje y el resto de las marcas estudiadas aquí son de dominio lingüístico. 
(9)

El otro día me explicaron científicamente la enfermedad que esta temporada padece el Real Madrid[...]. [...] el Real Madrid padece claustrofobia cuando juega en casa [...] y agorafobia cuando sale fuera (J. Cueto, El País Semanal, 28-XI-2004).

En (9) vemos cómo la palabra científicamente nos pone en alerta sobre la posibilidad de una interpretación irónica del enunciado, y efectivamente, a continuación nuestra sospecha se confirma por la aparición de dos términos psiquiátricos en cursiva. Se trata, en palabras del propio autor, de "aplicar al partido del domingo teorías relacionadas con la psiquiatría colectiva de los desarreglos emocionales del cerebro", con una intención claramente irónica. Algunas palabras de alerta se emplean con tanta frecuencia que parecen asociar el significado irónico antes que el habitual. Éste podría ser el caso, por ejemplo, de algunos adjetivos en grado superlativo: doctísimo, dignísimo, guapísimo, inteligentísimo, etc.

Otro procedimiento que nos puede indicar la presencia de la ironía es la reinterpretación de unidades fraseológicas, como se ve en el siguiente fragmento:

No puedo entender que no quede en ti ni siquiera una brizna, un suspiro, un mensaje, procedente de aquella época en que, según me has dicho, te postrabas ante un Sagrado Corazón de Jesús sangrante y le pedías que les diera morcilla a todas las niñas delgadas de tu barrio (M.Torres, El País Semanal, 5-XII-2004).

En (11) podemos observar cómo la desautomatización ${ }^{12}$ de una unidad fraseológica da lugar a la ironía. Si sustituyéramos la palabra morcilla por pan, por ejemplo, el enunciado perdería la ecoicidad que nos remite a la locución darle morcilla a alguien y, por consiguiente, no conseguiríamos el efecto irónico.

Las repeticiones, que mencionan explícitamente algo dicho, marcan evidentemente la ironía. Conviene distinguir aquí dos tipos de repeticiones. En el primer tipo, el hablante simplemente repite total o parcialmente el enunciado de su interlocutor, lo que provoca el efecto irónico. Por ejemplo:

\section{(11)}

- Entonces yo, como no soy tonta, cojo el teléfono y llamo directamente a su novio. Y ahora ninguno de los dos me habla.

- Mujer, tú que no eres tonta, podías haber pensado que esto iba a pasar.

El segundo tipo de repeticiones se refiere a la aparición iterativa de un enunciado a lo largo del mismo texto. Dicha reiteración nos hace sospechar que la intención del autor es distinta a la expresada por el enunciado. Schoentjes cita como ejemplo un fragmento de Julio César de William Shakespeare. Se trata del elogio fúnebre que pronuncia Marco Antonio y en el que aparece no menos de cuatro veces la frase "Bruto es un hombre honrado". Conocemos la verdadera historia de la muerte de César, así que la repetición funciona aquí como indicador para el público de Marco Antonio, no para el de Shakespeare.

12 Sobre el fenómeno de la desautomatización en unidades fraseológicas, véanse en especial Zuluaga (1980) y Ruiz Gurillo (1997). 
A diferencia de los indicadores vistos hasta ahora, en palabras de Schoentjes (2003: 148), existen figuras específicas ligadas desde siempre a la ironía. Entre tales figuras podemos destacar la lítotes, la hipérbole y el oxímoron.

Con la lítotes se da a entender más de lo que se dice, y es en este punto donde esta figura confluye con la ironía. La atenuación del pensamiento que caracteriza a la lítotes está en la base de las ironías que presentan los hechos relevantes como algo sin importancia, como muestra (12):

¿Qué lo del Prestige fue peor de lo que pudo ser por la incompetencia de los ministros Cascos y Rajoy? Nada, eso es pasado. ¿Qué todos los despropósitos de Yak y la posterior y dolosa chapuza con la identificación de los cuerpos se debieron a la frivolidad de Trillo y a la tacañería de Aznar? No me vengan con historias antediluvianas. ¿Qué la participación de España en la Guerra de Irak fue una despreciativa tozudez de Aznar, en contra de la mayoría de los españoles, y además un error, y además una decisión sustentada sólo por mentiras? Déjense de eso, la actualidad manda (J. Marías, El País Semanal, 28-XI-2004).

A diferencia de la lítotes, la hipérbole consiste en exagerar la verdad, lo que también puede ocasionar un efecto irónico. Ya hemos hablado de adjetivos en grado superlativo, cuyo uso frecuente ha provocado que hayan adquirido un fuerte matiz irónico. En (13) también podemos ver cómo la presencia de elementos hiperbólicos nos hace sospechar cierto tono irónico del enunciado:

Los dirigentes del PSOE, sus militantes y votantes tienen sobradas razones para estar crispados, tensos, rígidos, en fin, al borde de un ataque de nervios (A. Maestre, "Comprensible crispación", Libertad Digital, 5-XII-2004).

El oxímoron, por último, consiste en combinar conceptos contradictorios. La ironía puede originarse mediante esta figura, como sucede en las expresiones de (14) y (15):

(14) La locuacidad de pez

(15) La fidelidad de Judas

\section{Conclusiones}

Como hemos intentado mostrar a lo largo de este artículo, el grupo GRIALE está elaborando una propuesta de estudio de la ironía verbal en español que se pueda aplicar a diversos géneros textuales. Somos conscientes de que nuestra labor se halla en sus inicios y que el camino que habremos de recorrer hasta aplicar los resultados a la enseñanza de español a extranjeros es todavía largo y difícil. Sin embargo, hemos apuntado en este artículo algunas ideas que permitirán avanzar:

- Los ejemplos analizados muestran que la explicación pragmática de la ironía como mención ecoica resulta más acertada que la observación del fenómeno como una figura retórica. No obstante, la tradición retórica ha llevado a cabo precisiones que resultan muy útiles para abordar este hecho pragmático desde una perspectiva más amplia. 
- Al margen de los constructos teóricos necesarios para comprender la ironía como un tipo de mención o metarrepresentación del que se hace eco el hablante ${ }^{13}$, resulta crucial considerar al menos tres tipos de contexto: el lingüístico, el situacional y el sociocultural ${ }^{14}$.

- La discriminación de los diversos contextos implicados en la ironía arroja luz sobre las circunstancias concretas de comunicación, esto es, sobre el canal oral u escrito sobre el que se genera ironía. Así, pues, se han observado al menos dos tipos de ironía que se han denominado ironía focalizada e ironía continuada. La primera se encuentra más relacionada con los contextos lingüístico y situacional y parece más propia de los textos orales. La segunda se encuentra relacionada con los contextos situacional y sociocultural y hasta el momento resulta más frecuente en los textos escritos. Ahora bien, tal correlación no se puede suponer definitiva y habremos de profundizar en ambas clases de ironía.

- Uno de los hechos más destacados es la aparición de diversos indicadores que contribuyen a interpretar este fenómeno. Los gestos o la mímica, el llamado tono irónico, los signos de puntuación, las palabras de alerta, la fraseología o ciertas figuras retóricas como la lítotes, la hipérbole o el oxímoron constituyen pistas para el destinatario. GRIALE va a seguir trabajando en tales indicadores, pues consideramos que en ellos puede encontrarse el punto de partida de nuestra aplicación didáctica.

- Por último, habremos de analizar con mayor detenimiento el papel del destinatario u oyente. Todos los trabajos afirman que sin él no hay ironía, por mucha elaboración que exista por parte del hablante. Entre las tareas pendientes se encuentra el establecimiento de una terminología adecuada que represente a ese interlocutor activo ${ }^{14}$ o la observación de los efectos causados por este en los diversos géneros textuales.

\section{Referencias bibliográficas}

Abelson, R. P. y R. C. Schank, (1987): Guiones, planes, metas y entendimiento. Barcelona, Paidós. Azorín, D. (Coord.) (2002): Alicante corpus de referencia. Alcore (Edición en CD-Rom).

Azorín, D. y J. L. Jiménez Ruiz (1999): Corpus oral de la variedad juvenil universitaria del español hablado en Alicante. Alicante, Instituto de Cultura Juan Gil Albert.

Bateson, J. (1972): Steps on a ecology of mind. Nueva York, Ballantine.

Briz, A. (Coord.) (Grupo Val.Es.Co.). (1995): La conversación coloquial. Materiales para su estudio. València, Universitat. Anejo XVI de Cuadernos de Filología.

Briz, A. y grupo Val.Es.Co. (2002): Corpus de conversaciones coloquiales. Madrid, Arco Libros (Anejo II de la revista Oralia).

Clark, H. y R. Gerrig (1984): “On the Pretense Theory of Irony", Journal of Experimental Psychology: General, 113, 1, págs. 121-126.

Culler, J. (1975): Structuralist Poetics. Londres, Routledge and Kegan Paul.

Dik, S. (1989): The Theory of Functional Grammar. Part 1: The Structure of the Clause. Dordrecht, Foris.

13 Veánse a este respecto los trabajos sobre la ironía desde la Teoria de la Relevancia: Wilson (2000), Gibbs (2000) y Noh (2000).

14 Sobre estos hechos, agradecemos las sugerencias del profesor Francisco Yus, sugerencias que el grupo GRIALE tendrá en cuenta en futuros análisis.

15 Sobre el oyente combatiente, véase en este mismo volumen Padilla (2004). 
Ducrot, O. (1982): Decir y no decir. Principios de semántica lingüistica. Barcelona, Anagrama (1 ed., 1972).

Ducrot, O. (1986): El decir y lo dicho. Polifonía de la enunciación, Barcelona, Paidós (1 ${ }^{a}$ ed., 1984).

Ducrot, O. (1988): Polifonía y argumentación. Cali, Universidad del Valle.

Ducrot, O. (1989): Logique, structure, énonciation. Paris, Minuit.

Ducrot, O. (1996): Slovenian Lectures / Conférences slovènes. Ljubljana, ISH.

Ducrot, O. (2003): "Quelques raisons de distinguer 'locuteurs' et "énonciateurs", Poliphonie, III: http://www.hum.ua.dk/romansk/Polyphonie_III/Oswald_Ducrot.htm (29-4-2003).

Ducrot, O. et alii (1980): Les mots du discours. Paris, Minuit.

Escandell Vidal, M.V. (1996): Introducción a la pragmática. Barcelona, Ariel.

Goffman, E. (1981): Forms of Talk. Philadelphia, University of Philadelphia Press.

Instituto Cervantes (2002): Marco de referencia europeo para el aprendizaje, la enseñanza y la evaluación de lenguas: http:/cvc.cervantes.es/obref/marco

Knox, N. (1961): The world irony and its context: 1500-1755. Durham (North Carolina), Duke University Press

Lausberg, H. (1980): Manual de Retórica literaria. 3 vols. Madrid, Gredos.

Muecke, D. (1970): Irony. Londres, Methuen.

Noh, E.-J. (2000): Metarepresentation. A Relevance-Theory Approach. Amsterdam, John Benjamins.

Padilla García, X. A. (2001): El orden de palabras en el español coloquial. Valencia, Universitat de València (tesis doctoral).

Padilla García, X. A. (2004): "Del oyente receptor al oyente combatiente", ELUA, 18.

Padilla García, X. A. (2005): Pragmática del orden de palabras. Alicante, Servicio de Publicaciones de la Universidad de Alicante.

Poyatos, F. (1994): La comunicación no verbal (I). Cultura, lenguaje y conversación. Madrid, Istmo. Quintiliano, M. F. (1997): Institutionis oratoriae: libri XII, 4 vols. Salamanca, Universidad Pontificia de Salamanca.

Reyes, G. (1994): Los procedimientos de cita: citas encubiertas y ecos. Madrid, Arco Libros.

Reyes, G. (2002): Metapragmática. Valladolid, Universidad.

Reyes, G.; E. Baena y E. Urios (2000): Ejercicios de pragmática. Madrid, Arco Libros (2 vols.).

Ruiz Gurillo, L. (1997): "Relevancia y fraseología: la desautomatización en la conversación coloquial”, Español Actual, 68, págs. 21-30.

Ruiz Gurillo, L. (en prensa): "La ironía verbal”. Hechos pragmáticos del español.

Schontjes, P. (2003): La poética de la ironia. Madrid, Cátedra

Sperber, D. (ed.) (2000): Metarepresentations. A multidisciplinary perspective. Oxford, University Press.

Sperber, D. y D. Wilson (1978): “Les ironies comme mentions" Poétique, 36, págs. 399-412

Torres Sánchez, Ma Á. (1997-1998): "Teorías lingüísticas del humor verbal”, Pragmalingüística, 5-6, págs. $435-448$.

Torres Sánchez, Ma Á. (1999): Aproximación pragmática a la ironía verbal. Cádiz, Universidad de Cádiz.

Torres Sánchez, Ma Á. (1999b): Estudio pragmático del humor verbal. Cádiz, Universidad de Cádiz. Utsumi, A. (2000): "Verbal irony as implicit display of ironic enviromment: Distinguishing ironic utterances from nonirony", Journal of Pragmatics, 32, págs. 1777-1806.

Van Dijk, T. (1977): Text and context. London, Longman.

Wilson, D. (2000): "Metarepresentation in linguistic communication." En Sperber, D. (ed.), págs. $411-448$.

Yule, G. (1996): Pragmatics. Oxford, Oxford University Press.

Zuluaga, A. (1980): Introduccción al estudio de las expresiones fijas. Tübingen, Max Hueber, Verlag. 